\title{
Power System Dynamic State Estimation based on Kalman Filter
}

\author{
Firas M. Taimah \\ Assistant Professor \\ Engineering College-Baghdad University
}

\author{
Akram N. Merzah \\ Ms.C. Student \\ Engineering College-Baghdad University
}

\begin{abstract}
This paper presented the application of Kalman Filtering technique in estimating the dynamic variables for the multimachine power systems. The Extended Kalman Filter (EKF) and Unscented Kalman Filter (UKF) are both appropriate tools to be applied in power system dynamic state estimation studies. EKF and UKF are implemented using a second-order swing equation and a classical generator model to estimate the dynamic state (generator rotor angle and generator rotor speed) and comparing the result which obtained from the two estimation algorithm (EKF and UKF) with the result from the fourth order Runge-Kutta method in order to show the statistical performance and estimation accuracy of each algorithm. The algorithms are mathematically demonstrated using the IEEE 14-bus test system. The results show that the UKF method gives an accurate performance in the dynamic state estimation for multi-machine power system than the EKFmethod. It gives minimum mismatch between estimated state and actual state.
\end{abstract}

\section{Keywords}

Dynamic State Estimation (DSE), Kalman Filter (KF), Extended Kalman Filter (EKF), Unscented Kalman Filter (UKF)

\section{INTRODUCTION}

The state estimation was first introduced and applied on the power system by Fred Schweppes in the 1970 [1]. State estimation represents the main part of energy management system which plays an essential role on the monitoring, control and stability analysis of electrical power system. State estimation can be classified into two types: static state and dynamic [2]. Static State Estimation (SSE) offers a snapshot of power system operating point reproduced by state variables [3].The state estimation process used the SCADA Data to evaluate the best estimate of the state of electrical power system. SCADA system receives the measurement through Remote Terminal Units (RTU) which gathered from the network. Measurement data include injected real power, reactive power, voltage magnitude and relative voltage phase angle of each bus bar [4]. Power system continuously vagaries with time but this change is slowly and steadily so that it is defined as quasi-static systems. This change occurred due to the load varying at buses and when the load varying, the generation feeding must be adjusted according to the load variation. As a result, the power flows and injections at the buses were changed; therefore, the power system is dynamic not static and the static state estimation fails to capture the nature state of power system [5].This lead to development Dynamic state estimation [6]. Dynamic state estimation (DSE) computes the state of the system by forming the time changing the behavior of power system the cater to dynamic variation of the power system and predict the state of the system at the next instant of time $(\mathrm{k}+1)$ [7]. Various techniques are available for state estimation which can be applied to the power system. Kalman filter is used to minimize the mismatch between the estimated states and the states [8]. The extended Kalman filter and unscented Kalman filter are sufficient techniques in the estimation of dynamic state vector element under transient condition.

\section{DYNAMIC STATE ESTIMATION}

A dynamic model is better than the static one in providing a good performance in monitoring system operating conditions, can be characterized by a process equation (1) and a measurement equation (2) as follows:

$X_{k+1}=f\left(k, X_{k}\right)+q_{k}$

$Z_{k}=h\left(k, X_{k}\right)+r_{k}$

where $k$ is the sample of time; $X_{k}$ is the state vector; $q_{k}$ denotes modeling uncertainties, corresponding to a white Gaussian noise with zero mean and covariance matrix $Q_{k} ; Z_{k}$ is the measurement vector; $h$ is a set of nonlinear load-flow functions for the current network configuration; $r_{k}$ is a Gaussian error vector, with zero mean and diagonal covariance matrix $R_{k}$

The simple idea of state estimation function is to find the best estimate of system state vector $\mathrm{X}$ of dynamic state variables:

$\mathrm{X}=\left[\omega_{i}, \delta_{i}\right]^{\mathrm{T}}$,

The measurement vector $\mathrm{Z}$ which gathered from the network by remote terminal units (RTU) are presented

$\mathrm{Z}=\left[\mathrm{V}, \theta, \mathrm{P}_{\text {inj }}, \mathrm{Q}_{\mathrm{inj}}\right]^{\mathrm{T}}$.

Static state estimation delivers only a snapshot of the present state vector (find $X_{k}$ at a certain time $k$ by using the set of measurement data received at the same instant of time $\mathrm{k}$ ).the aims of the dynamic state estimation is to offer not only timevarying behavior of the system but also expect the future state of the system[9].

\subsection{Extended Kalman Filter}

One of the greatest used techniques in power system dynamic state estimation is Extended Kalman Filter (EKF) that takes into its accounts both the entering measurements and the expected state to get an optimal estimate of the system state [9]. Kalman filter is appropriate for real-time estimation since the estimation is finished for any instantaneous time. EKF is a discrete Kalman filter adapting to nonlinear system estimation through linearization.

For a nonlinear dynamic system defined by differential algebraic functions (DAEs) in (3) and further in discrete form 
in (4), the aim of EKF is to reduce the covariance of the incompatibility between the estimated states and the states.

$$
\begin{gathered}
\frac{d y}{d x}=f(x, y, u, w) \\
0=g_{c}(x, y, u, v)
\end{gathered}
$$

Where the $x$ vector represents the state variables, the $y$ vector represents the algebraic variables, $u$ is the vector of input variables, $w$ and $v$ are processing noise and measurement noise. The subscript "c" represents the continuous form. The discrete form of (1) is shown below:

$$
\begin{aligned}
X_{k} & =X_{k-1}+f_{c}\left(X_{k-1}, u_{k-1}, w_{K-1}\right) \Delta \mathrm{t} \\
& =f\left(X_{k-1}, u_{k-1}, w_{K-1}\right) \\
y_{k} & =h\left(X_{k}, u_{k}, v_{K}\right)
\end{aligned}
$$

The EKF problem can be resolved in a two-step [10]

1) Step 1 -predicted state

$$
\begin{gathered}
\hat{X}_{k}^{-}=f\left(\hat{X}_{k-1}, h\left(\hat{X}_{k-1}, u_{k-1}\right), u_{k-1}, 0\right) \\
P_{k}^{-}=A_{k} P_{k-1} A_{k}^{T}+W_{k} Q_{k-1} W_{k}^{T}
\end{gathered}
$$

\section{2) Step 2-corrected state}

$$
\begin{aligned}
& K_{k}=P_{k}^{-} H_{k}^{T}\left(H_{k} P_{k}^{-} H_{k}^{T}+V_{k} R_{k} V_{k}^{T}\right) \\
& \hat{X}_{k}=\hat{X}_{k}^{-}+K_{k}\left(Z_{k}-h\left(\hat{X}_{k}^{-}, u_{k}, 0\right)\right) \\
& P_{k}=\left(I-K_{k} H_{k}\right) P_{k}^{-}
\end{aligned}
$$

where "-" means a priori state, $A_{k}$ and $W_{k}$ are the Jacobians process at step $\mathrm{k}, P_{k}$ is a covariance matrix of the state estimation error and is also named gain factor matrix, and $Q_{k}$ is the process noise covariance at step k. $H_{k}$ and $V_{k}$ are the measurement Jacobians at step $\mathrm{k}$, and $R_{k}$ is the measurement noise covariance at step $\mathrm{k}$.

$$
A=\frac{\partial f}{\partial x}, H=\frac{\partial h}{\partial x},=\frac{\partial f}{\partial W}, V=\frac{\partial h}{\partial V}
$$

\subsection{Unscented Kalman Filter}

The UKF is a proficient discrete-time recursive filter capable of solving estimation issues which are having the formula shown below

$X_{k}=f\left(X_{k-1}, k-1\right)+q_{k-1}$

$y_{k}=h\left(X_{k}, k\right)+r_{k}$

Where $X$ is the state vector, $y$ is the measurement vector, while $q_{k-1}$ and $r_{k}$ are, respectively, the system and measurement Gaussian noises with zero mean and uncorrelated covariance matrices $\mathrm{Q}$ and $\mathrm{R}$, respectively. Functions $f$ and $h$ are non-linear functions demonstrating the system and measurements models in terms of the state variables and other system inputs.

The UKF merges the classical Kalman filter idea and the UT [11]. The prime benefit of the UKF over the EKF is the fact that (8) and (9) were not approximated, so (8) and (9) were not linearized; which averts missing of higher order information and therefore enhances the features of the estimator. Moreover, not any Jacobian or Hessian matrices are required, which decreases the CPU necessities and submits computational improvements over the EKF. The UKF will provide higher precision than the EKF by way of higher order relations of the non-linear model functions are taken into consideration [11]. The UKF involves three main steps which are demonstrated below:

\section{Step 1: sigma points calculation}

It is required to generate sets of $2 n+1$ sigma points [12], by means of the state vector $x$ at time $k-1$ and the equivalent covariance matrix $P_{k-1}$

$X_{k-1}=\left[\begin{array}{lll}x_{k-1} \ldots x_{k-1}\end{array}\right]+\sqrt{c}\left[\begin{array}{lll}0 & \sqrt{P_{k-1}} & -\sqrt{P_{k-1}}\end{array}\right]$

Where $c=n+\lambda$ and $\lambda=\alpha^{2}(n+\kappa)-n, \lambda$ is a scaling parameter with the propagated constant $\left(10^{-4} \leq \alpha \leq 1\right)$ which proposed for Gaussian distribution [13] , the scaling parameter $\kappa$ can be taken $\kappa=3$ or 0 .In fact, The purpose of used parameter $\kappa$ is to decrease the high order error of approximation [14]. For the determination of the estimation initialization (i.e. when $\mathrm{k}=0$ ), the initial state vector also the initial covariance matrix must be clear beforehand according to a priori familiarity of the system.

Step 2: Kalman filter state prediction

Calculate, one by one, the series of sigma points determined in phase 1 through the state update function

$\hat{X}_{k}^{i}=f\left(X_{k-1}^{i}, k-1\right) i=0, \ldots \ldots, 2 n$

Where $X_{k-1}^{i}$ is the $(i+1)$ th column of matrix $X_{k-1}$ and the resultant $\hat{X}_{k}$ is a $n \times(2 n+1)$ matrix holding the generated sigma points. Then, calculate the expected state mean vector $x_{k}^{-}$and the expected covariance matrix $P_{k}^{-}$as follows

$x_{k}^{-}=$

$\sum_{i=0}^{2 n} W_{i}^{m} \hat{X}_{k}^{i}$

$P_{k}^{-}=\sum_{i=0}^{2 n} W_{i}^{c}\left[\left(\hat{X}_{k}^{i}-X_{k}^{-}\right)\left(\hat{X}_{k}^{i}-X_{k}^{-}\right)^{T}\right]+Q_{k-1}$

The weight factors in expression (11) and (12) is evaluated by the following Formulae

$W_{0}^{m}=\frac{\lambda}{n+\lambda}$

$W_{0}^{c}=\frac{\lambda}{n+\lambda}+\left(1-\alpha^{2}+\beta\right)$

$W_{i}^{m}=W_{i}^{c}=\frac{1}{2(n+\lambda)}$

Where the variable $\beta$ can be taken a value of two ( $\beta=2$, the optimum value of variable $\beta$ for Gaussain distribution function is two [12]).

Step 3: Kalman filter state correction

Compute the sigma points matching to the expected state mean vector and the covariance matrix

$$
X_{k}^{-}=\left[\begin{array}{lll}
x_{k}^{-} \ldots & x_{k}^{-}
\end{array}\right]+\sqrt{c}\left[\begin{array}{lll}
0 & \sqrt{P_{k}^{-}} & -\sqrt{P_{k}^{-}}
\end{array}\right]
$$

Propagate the new sigma points obtained from equation (15) through the measurement update function 
$Y_{k}^{-}=h\left(X_{k}^{-}, k\right)$

The mean of the propagate new points is computed from the following expression

$\mu_{k}=\sum_{i=0}^{2 n} W_{i}^{m} Y_{k}^{-i}$

Calculate the measurement covariance matrix. In addition, the cross-covariance of the state and measurement:

$S_{k}=\sum_{i=0}^{2 n} W_{i}^{c}\left[\left(Y_{k}^{-i}-\mu_{k}\right)\left(Y_{k}^{-i}-\mu_{k}\right)^{T}\right]+R_{k}$

$C_{k}=\sum_{i=0}^{2 n} W_{i}^{c}\left[\left(X_{k}^{-i}-x_{k}^{-}\right)\left(Y_{k}^{-i}-\mu_{k}\right)^{T}\right]$

Then, the filter's gain matrix $K_{k}$ is obtained as the following

$K_{K}=C_{k} S_{k}^{-1}$

The correction state vector mean and its corresponding covariance are evaluated by the the following expression

$x_{k}=x_{k}^{-}+K_{K}\left[y_{k}-\mu_{k}\right]$

$P_{k}=P_{k}^{-}-K_{K} S_{k} K_{k}^{T}$

\section{CASE STUDY}

Both extended Kalman filter and unscented Kalman filter have benefits and disadvantages so that it is important to investigate a specific case in an electrical power system for comparison between them. In this section, the algorithms presented in section 2 were tested by using IEEE 5- generator, 14-bus test system showing in Figure 1 [15]. A three-phase fault happens on the line between bus 3 and bus 4 at time $t=2$ $\mathrm{s}$. the fault is cleared after $0.1 \mathrm{~s}$ and line 3-4 is removed. The simulation time period is set as $\Delta \mathrm{t}=0.02 \mathrm{~s}$.

To compare the accuracy of estimation of each filtering algorithm, the estimation error was evaluated by using the following expression

$\xi_{k}=\frac{1}{2 n} \sum_{i=0}^{2 n}\left|X_{k}^{i}-\hat{X}_{k}^{i}\right|$

Where $X$ is denoted the actual state which evaluated from numerical integration method of transient stability analysis and $\hat{X}$ is denoted the estimated state vector which calculated from the Kalman filter algorithm, $2 n$ is the number of states ( $n$ state for generator rotor speed and $n$ state for generator rotor angle)

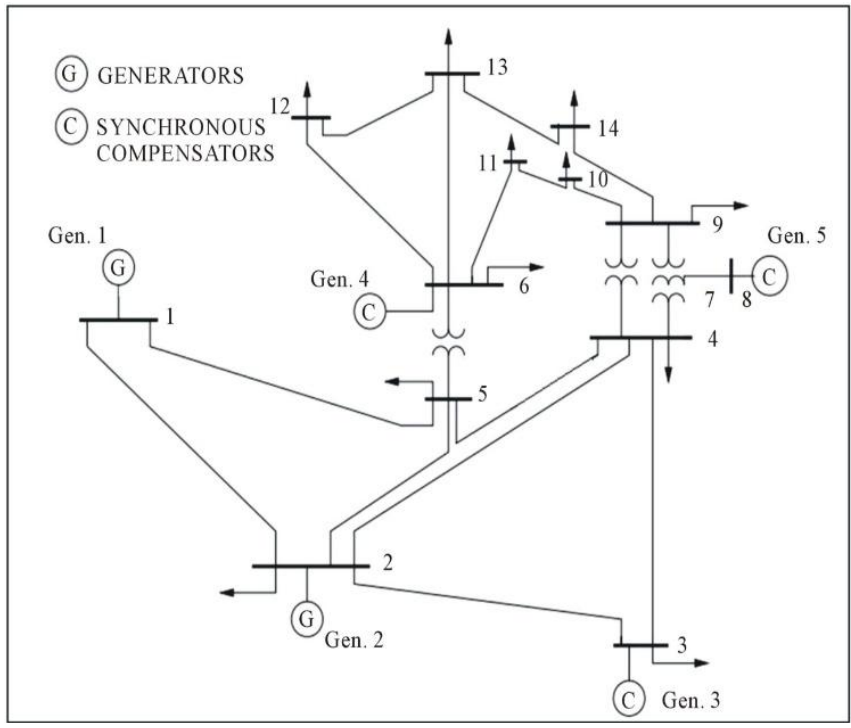

Fig 1: The single line diagram of the IEEE 5-generator 14bus test system

And the overall estimation error is calculated by the following formula

$\xi=\frac{1}{k_{\max }} \sum_{k=1}^{k_{\max }} \xi_{k}$

Figure 2 shows the actual value of rotor speed for Gen.1 and its estimated value by using both the EKF and UKF algorithms. Figure $2 \mathrm{a}$ shows the plot of the rotor speed $\omega_{1}$ estimated by EKF and Figure 2b by UKF. Figure 3 shows the plots of rotor speed for Gen. 2 using EKF as shown on Figure $3 \mathrm{a}$ and UKF shown Figure 3b, Figure 4 shows the relative rotor angle for Gen. 2 estimated by both EKF as shown on Figure $4 \mathrm{a}$ and UKF on Figure $4 \mathrm{~b}$.

The comparison between the EKF and UKF according to performance indices and estimation error is demonstrated in Table 1

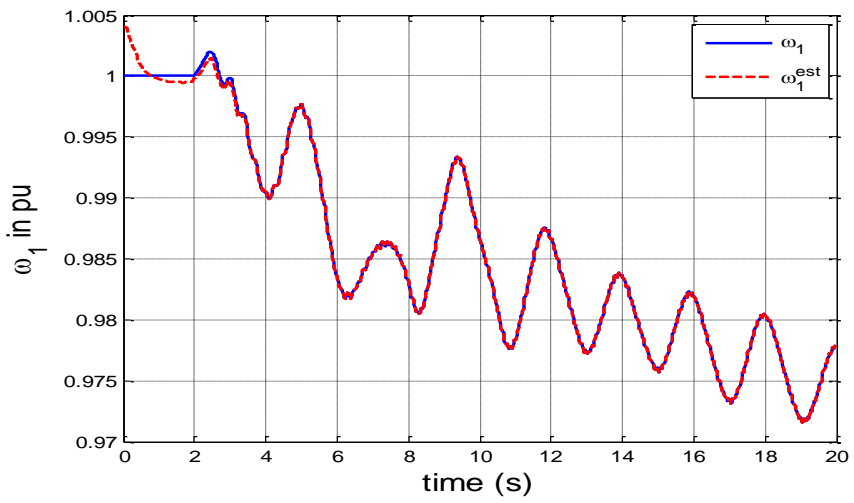

Fig 2a: Estimation of Rotor Speed for Gen. 1 by EKF 


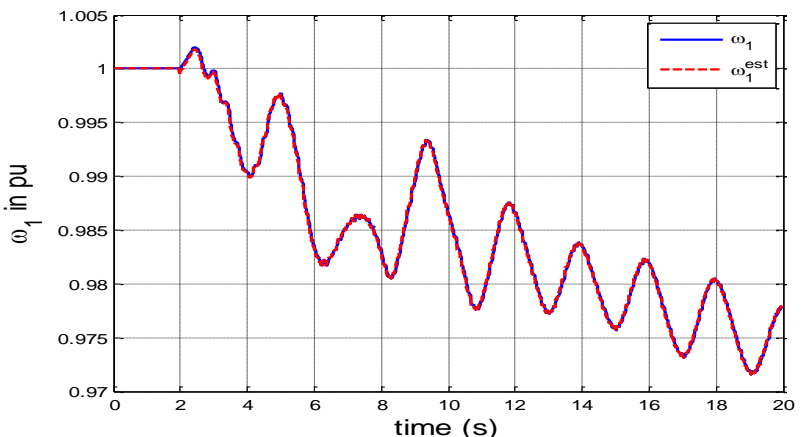

Fig 2b: estimation of rotor speed for Gen. 1 by UKF

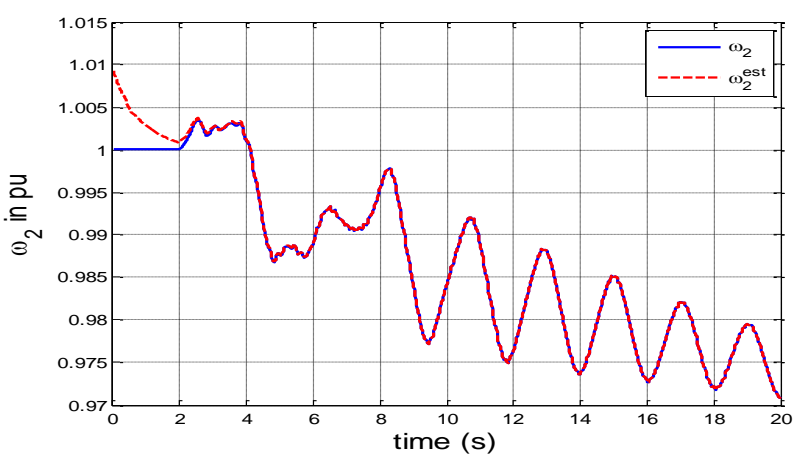

Fig 3a: Estimation rotor speed for Gen. 2 by EKF

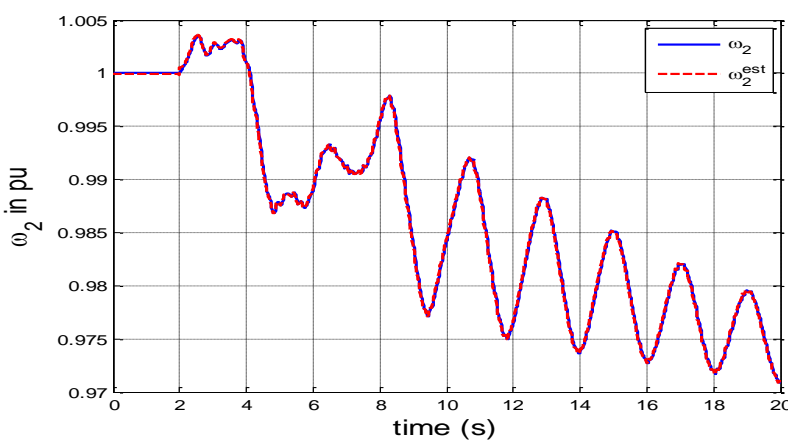

Fig 3b: Estimation rotor speed for Gen. 2 by UKF

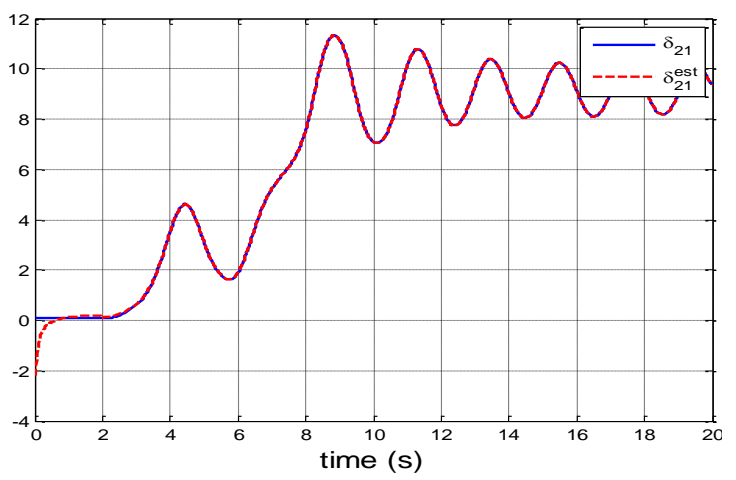

Fig 4a: Estimation of relative rotor angle for Gen. 2 by EKF

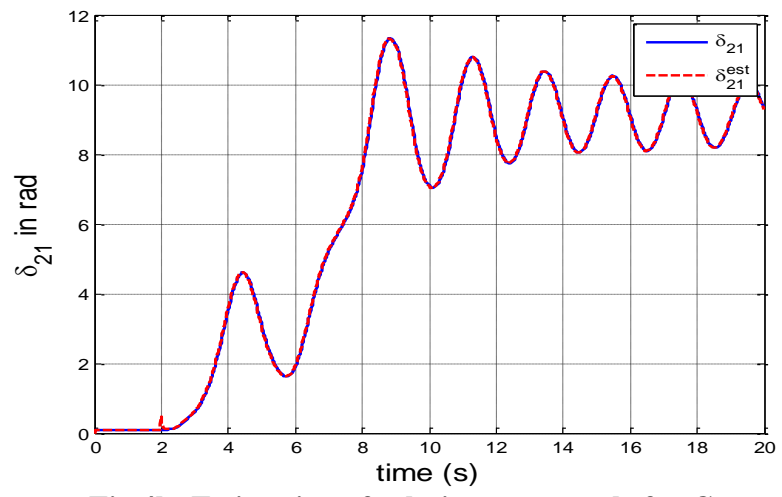

Fig 4b: Estimation of relative rotor angle for Gen. 2 by UKF

Table 1. Comparison of performance indices of EKF and UKF

\begin{tabular}{|c|c|c|}
\hline Performance Index & EKF & UKF \\
\hline$\xi$ & 0.0109 & 0.0082 \\
\hline$\xi_{\omega}$ & $4.1251 \times 10^{-4}$ & $2.3028 \times 10^{-4}$ \\
\hline$\xi_{\delta}$ & 0.0236 & 0.0162 \\
\hline
\end{tabular}

\section{CONCLUSION AND DISCUSSIONS}

The Dynamic State Estimation (DSE) is used to evaluate the state of the system by modeling the time changing the behavior of the system; therefore, it offers a most important role in monitoring, stability analysis and control of electrical power system. This paper proposed two methods (EKF and UKF) which offer an accurate performance in the dynamic state estimation for the multi-machine power system. As checked by the simulation result, the UKF method is very promising technique compared to EKF method as it is more efficient and the computational demand is lower than EKF. Because of the UKF no need to obtain any partial derivative or Jacobian computation, the implementation of UKF is easier than EKF and it provides an efficient estimation method for the non-linear system. AS seen in Table 1 that illustrates the comparison between the values of the estimation error of EKF and UKF method, UKF gives minimum mismatch between the actual state and estimated state and it provides more accurate performance.

For the future work, the dynamic parameters, other than rotor speed and rotor angle, of the generators can also be considered in the dynamic state estimation and the dynamic state vector can be extended. Also the work can be expanded not only operates under normal operating conditions but involve many cases, like: when bad data is available (to see the ability of the state estimation in handling bad data), when there is topology error condition.

\section{REFERENCES}

[1] Fred. C. Schweppe, J. Wildes, and Douglas. B. Rom, "Power system static-state estimation, part I, II and III", IEEE Transactions on Power Apparatus and Systems, vol. PAS-89, NO. 1, pp. 120-135, 1970.

[2] Ankhi Gulati , Niyati Gulati and Sushil Kr. Solanki, " Overview of State Estimation Technique for Power System Control", IOSR Journal of Electrical and 
Electronics Engineering, vol.8, pp.51-55, Nov. - Dec. 2013.

[3] F. Schweppe, "Power system static-state estimation, part III: Implementation", IEEE Trans. Power App. Syst., vol. PAS-89, no. 1, pp. 130-135, 1970.

[4] Sideig A. Dowi,and Amar Ibrahim Hamza, "Dynamic State Forecasting in Electric Power Networks", Journal of Power and Energy Engineering, vol. 2, pp. 1-11, 2014.

[5] Omprakash s. mankar and K. Vadirajacharya, "Kalman filterI anlysis in dynamic state of power system". International Research Journal of Engineering and Technology, vol. 2, pp.1170-1173, 2015.

[6] A. S. Debs and R. E. Larson, "A Dynamic Estimator for Tracking the State of a Power System", IEEE Transactions on Power Apparatus and Systems, vol. PAS-89, no. 7, pp. 1670-1678, 1970.

[7] Amit Jain and Shivakumar N. R., "Power System Tracking and Dynamic State Estimation", In IEEE PES Power Systems Conference Exposition (PSCE) 2009.

[8] K. P. Schneider, Z. Huang, B. Yang, M. Hauer, and Y. Nieplocha, "Dynamic state estimation utilizing high performance computing methods," Power Systems Conference and Exposition, 2009. PSCE '09. IEEE/PES. pp. 1-6, 2009.
[9] C. Bila, "Power system dynamic state estimation and load modeling", M.S. thesis, Dept. Electrical and Computer Eng., Northeastern Univ., Boston, Massachusetts, 2014

[10] D. Simon, “Optimal State Estimation”, WileyInterscience, Jone Wiley and Sons, Inc., Hoboken, New Jersey, 2006.

[11] G. Valverde and V. Terzija, "Unscented Kalman filter for power system dynamic state estimation", IET Generation, Transmission and Distribution, vol.5, Iss. 1, pp. 29-37, July 2010.

[12] Huang, Li and Yan W., "Estimating parameters of synchronous generators using square-root unscented Kalman filter”, Electric Power Syst. Res., vol. 8, no. 9, pp. 1137-1144, 2010.

[13] Sarkka, S.: "On unscented Kalman filtering for state estimation of continuous-time nonlinear systems", IEEE Trans. Autom. Control , vol.52, (9), pp. 1631-1641, 2007.

[14] D. Simon, "Optimal state estimation: Kalman, H infinity, and nonlinear approaches", Wiley, New Jersey, 2006.

[15] Dabbagchi, I.and Christie R., "Power system test case archive", (Resources at Electrical Engineering, The University of Washington, 1993). 